\title{
Nephron Supply Is a Major Determinant of Long-Term Renal Allograft Outcome in Rats
}

\author{
Harald S. Mackenzie, ${ }^{\star \ddagger}$ Stefan G. Tullius, ${ }^{5}$ Uwe W. Heemann, ${ }^{5}$ Haruhito Azuma, ${ }^{5}$ Helmut G. Rennke," Barry M. Brenner, ${ }^{\star \ddagger}$ \\ and Nicholas L. Tilney $\$$ \\ * Renal Division, Department of Medicine, and Departments of "Pathology and \$ Surgery, Brigham and Women's Hospital; \\ and $^{\ddagger}$ the Harvard Center for the Study of Kidney Diseases, and ${ }^{\S}$ Surgical Research Laboratories, Harvard Medical School, \\ Boston, Massachusetts 02115
}

\begin{abstract}
The effects of augmenting the nephron supply on indices of allograft injury were assessed in a rat model of "chronic rejection." Orthotopic renal allotransplantation into uninephrectomized rats was followed by excision (allograft-alone group) or preservation of the remaining native kidney (allograft + native kidney group) such that the total kidney complement was either the allograft alone, or the allograft plus one retained native kidney. After $18 \mathrm{wk}$, values for GFR $(1.85 \pm 0.3 \mathrm{ml} / \mathrm{min})$ and kidney weights $(2.3 \pm 0.2 \mathrm{~g})$ in allograft-alone rats were far in excess of corresponding values in the allograft of allograft+native kidney rats $(0.88 \pm 0.1$ $\mathrm{ml} / \mathrm{min}$ and $1.1 \pm 0.5 \mathrm{~g}$, respectively). Proteinuria $(35 \pm 2$ $\mathrm{mg} / \mathrm{d})$ and allograft glomerulosclerosis $(24 \pm 8 \%)$ also characterized allograft-alone but not allograft+native kidney rats, in whom glomerular structure (allograft glomerulosclerosis, $4 \pm 1 \%$; native kidney glomerulosclerosis, $0 \%$ ) and glomerular functional integrity (proteinuria $7 \pm 0.7 \mathrm{mg} / \mathrm{d}$ ) were well preserved. Thus, the observed allograft protection derived from the presence of a retained recipient native kidney supports the hypothesis that a single renal allograft contains insufficient nephrons to prevent progressive renal injury, implicating nephron supply as a major determinant of long-term allograft outcome. (J. Clin. Invest. 1994. 94:2148-2152.) Key words: chronic rejection - transplantation • kidney diseases $\cdot$ glomerular filtration rate $\cdot$ glomerulosclerosis
\end{abstract}

\section{Introduction}

Gradual deterioration of renal transplant function, attributed to an ill-defined process termed "chronic rejection," accounts for nearly all cases of late renal allograft failure in humans, and is currently a major impediment to the long-term success of renal

Address correspondence to Harald S. Mackenzie, Renal Division, Department of Medicine, Brigham and Women's Hospital, 75 Francis Street, Boston, MA 02115. 1994.

Received for publication 28 April 1994 and in revised form 28 July

J. Clin. Invest.

(C) The American Society for Clinical Investigation, Inc.

$0021-9738 / 94 / 11 / 2148 / 05 \$ 2.00$

Volume 94, November 1994, 2148-2152 transplantation (1). In contrast to the striking improvements in 12-mo renal allograft survival rates made over recent years, late organ failure rates have remained static throughout the entire transplant experience (2), apparently unaffected by the introduction of better immunosuppressive regimes. Without slowing the rate of late allograft loss, only $40 \%$ of new allografts from cadaver donors can be expected to survive at $8.5 \mathrm{yr}$ after transplantation $(2,3)$. To explore possible factors underlying late renal allograft loss we have conducted studies in a rat model of chronic renal allograft failure. The Fisher $344 \rightarrow$ Lewis rat model of long-surviving renal transplantation, first described by White et al. (4), and recently adapted by Diamond et al. (5), is considered to be a useful model of "chronic rejection." As in humans (6-8), striking similarities are evident between the natural history and histopathological appearances of late allograft failure in F344 $\rightarrow$ Lew rats and those shared by a variety of other conditions also characterized by progressive kidney failure, e.g., hypertensive nephropathy or extensive surgical ablation of renal mass $(4,5,9,10)$. Over the past $15 \mathrm{yr}$, extensive experimental study of rats with renal mass ablation has given rise to a unifying theory for the progressive nature of renal disease in conditions such as those listed above (9). A selfperpetuating cycle of nephron loss is proposed with glomerulosclerosis as the eventual consequence of the compensatory renal hemodynamic responses to inadequate nephron mass (11). As the allograft is a single kidney facing the workload of two, but with a rapidly diminishing pool of functioning nephrons, due to early losses from ischemia-reperfusion injury, acute rejection, and cyclosporine nephrotoxicity, it may be likened to the remnant kidney in experimental models where extensive ablation of renal mass initiates a rapidly progressive course of hemodynamically mediated glomerular injury. We propose that a hemodynamically mediated cycle of progressive injury in the solitary allograft is an inevitable consequence of inadequate numbers of functioning nephrons surviving the acute sequelae of transplantation (12). Thus, we postulate that supplementing the total numbers of nephrons in the F344 $\rightarrow$ Lew rat model will slow the tempo of progressive injury affecting the renal allograft by elevating the nephron supply above a critical point beneath which accelerated, progressive nephron injury is likely to occur.

To test this hypothesis we assessed indices of allograft (A) ${ }^{1}$

1. Abbreviations used in this paper: A, allograft; A-alone, allograft alone; $A+N K$, allograft plus native kidney; GFR, glomerular filtration rate; ERPF, effective renal plasma flow; PAH, para-amino hippurate; F344, Fisher 344 rat; F344 $\rightarrow$ Lew, Fisher 344 to Lewis renal transplantation; SBP, systolic blood pressure; FSGS, focal and segmental glomerulosclerosis and hyalinosis. 
function and allograft injury in two groups of uninephrectomized rats prepared either with (A-alone), or without $(\mathrm{A}+$ NK) subsequent excision of the contralateral native kidney (NK) after orthotopic transplantation of a Fisher 344 kidney to a Lewis recipient. Thus A-alone rats had a total kidney complement of one, whereas $A+N K$ rats had a total complement of two: the allograft and one retained native kidney. This paradigm allows comparison of allograft injury between one group of rats $(A+N K)$ having approximately twice the initial total nephron supply of the other (A-alone); yet histocompatibility differences between each group and the total alloantigen load presented to the recipients are kept constant.

\section{Methods}

Kidney grafting. Under ether anesthesia, the left kidney of each male F344 donor rat was isolated, excised, and cooled. The kidneys were then positioned orthotopically in anesthetized, weight-matched Lewis recipients whose left renal vessels had been mobilized, clamped, and the native kidney excised. The donor and recipient renal artery, vein and ureter were each then anastomosed end-to-end with 10-0 prolene sutures, without the use of ureteral stenting. The contralateral native kidney was either excised on the 10th post-operative day (A-alone group, $n=15$ ) or preserved (A $+\mathrm{NK}$ group, $n=7$ ). All rats were housed under standard conditions and received standard rodent diet containing $0.48 \% \mathrm{Na}$. All rats were treated in accordance with institutional guidelines for animal welfare. Serial measurements of systolic blood pressure (SBP) were carried out monthly using the tail-cuff method; urinary protein excretion rates, determined by precipitation with sulfosalicylic acid, were also determined monthly from $24 \mathrm{~h}$ urine collections from individual rats in metabolic cages.

After 18 wk, clearance studies were carried out. Anesthesia was induced with Brevital (Eli Lilly, Indianapolis, IN), 0.2-0.3 ml, and maintained with Inactin (Byk-Gulden, Konstanz, Germany), $0.1 \mathrm{ml} /$ $100 \mathrm{~g}$ body weight, i.v. After tracheostomy, cannulas were placed in the femoral vein for the administration of fluids and inactin, and in the femoral artery for the continuous measurement of blood pressure and intermittent blood sampling. Mean arterial blood pressure was measured by a Gould transducer and the output displayed on a chart recorder. Bovine salt-poor albumin ( $4 \%$ ) in $0.9 \%$ saline, equivalent to $0.5 \%$ body weight, was infused at $100 \mu \mathrm{l} / \mathrm{min}$ to replace fluid and protein losses incurred during surgery. Inulin ( $7.5 \%$ ) and $0.75 \%$ para-amino hippurate (PAH) in $0.9 \%$ saline were also infused at $20 \mu \mathrm{l} / \mathrm{min}$ throughout the experiments. The allograft was exposed through a midline incision and the ureter catheterized. Where required, the right ureter or the bladder was catheterized to allow collection of urine from the native kidney. After a 60-min stabilization period, two 20-min urine samples were obtained with blood sampling at the midpoint of each clearance period. Urine volumes were determined gravimetrically. Concentrations of inulin in blood and plasma were determined by an anthrone method; concentrations of PAH were determined by a colorimetric method. In three A-alone rats, clearances could not be obtained for technical reasons. A further three rats in this group were also excluded from study due to the presence of moderate hydronephrosis. A small number of A-alone $(n=5)$ and $\mathrm{A}+\mathrm{NK}(n=5)$ rats were available for study of proteinuria $1 \mathrm{yr}$ after transplantation.

After the clearance studies, the kidneys were removed, fixed in phosphate-buffered formalin (10\%), stained using the Periodic AcidSchiff method, and prepared for histopathologic evaluation. The frequency of focal and segmental sclerosis and hyalinosis was determined by examining all glomerular profiles contained within one or two coronal sections from each kidney such that on average $154 \pm 22$ glomeruli were counted for each kidney (range: 54-400). The frequency of glomeruli with focal and segmental sclerotic lesions was then expressed as a percentage of the total number of glomeruli counted.

All data are presented as mean \pm SEM. Statistical comparisons were

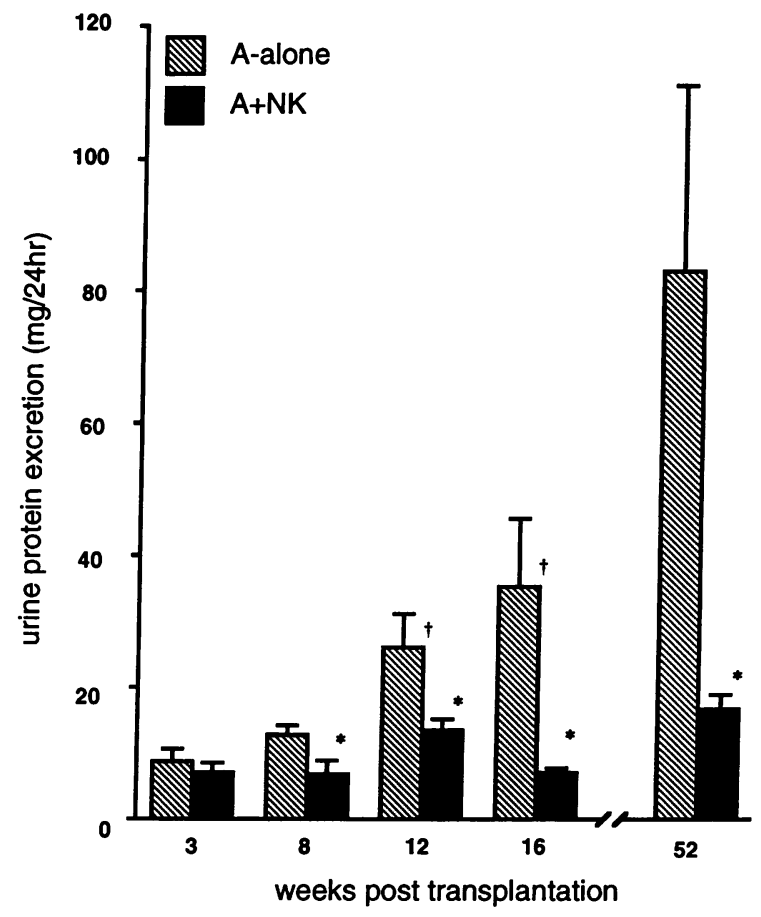

Figure 1. Urinary protein excretion from the solitary allograft (A-alone, $n=9$ ) is depicted by the shaded columns and from rats in the A+NK group $(n=7)$ by the solid columns. At $12 \mathrm{wk}, \mathrm{A}$-alone protein excretion rates became significantly greater than at week 3 , at which point values for $\mathrm{A}+\mathrm{NK}$ and $\mathrm{A}$-alone were similar. Beyond week 3, UprotV was significantly lower in A+NK than in A-alone rats. Data at 52 wk are from separate groups of identically prepared F344 $\rightarrow$ Lew rats. $\left({ }^{\dagger} P\right.$ $<0.05$ vs. week 3 ; $^{*} P<0.05$ vs. A-alone by ANOVA and Sheffé's test).

made using $t$ tests for unpaired data, or analysis of variance followed by Scheffé's test, as appropriate (13).

\section{Results}

The evolution of proteinuria is shown in Fig. 1 and, in the Aalone group, is characteristic of the pattern previously reported for this model (5). Over $16 \mathrm{wk}$, protein excretion in the Aalone group increased progressively, a trend also evident in those few rats surviving at $1 \mathrm{yr}$. Proteinuria indicates loss of the integrity of the glomerular filtration barrier and has previously been associated with the presence of focal glomerulosclerosis in F344 $\rightarrow$ Lew rats with solitary allografts (5), and in rats with experimental renal mass ablation (11). In contrast, the level of protein excretion from the $\mathrm{A}+\mathrm{NK}$ rats remained at near-normal levels, even in those rats studied at one year (Fig. 1). SBP, while tending to be greater in the A-alone group when compared with $A+N K$ rats, did not show a trend towards progressive increase; rather SBP remained at somewhat elevated levels throughout the study period, without attaining overtly hypertensive values (Fig. 2). Under anesthesia, mean arterial pressure for the A-alone group was $114 \pm 8 \mathrm{mmHg}$, numerically higher but not significantly different to that for the A $+\mathrm{NK}$ group, 103 \pm 6 . From similar body weights for A-alone (254 \pm 9 $\mathrm{g})$ and $\mathrm{A}+\mathrm{NK}$ recipients $(242 \pm 17 \mathrm{~g})$ at the time of transplantation, rats grew at similar rates reaching comparable body weights at the time of the acute studies: $382 \pm 16 \mathrm{~g}$ for A-alone 


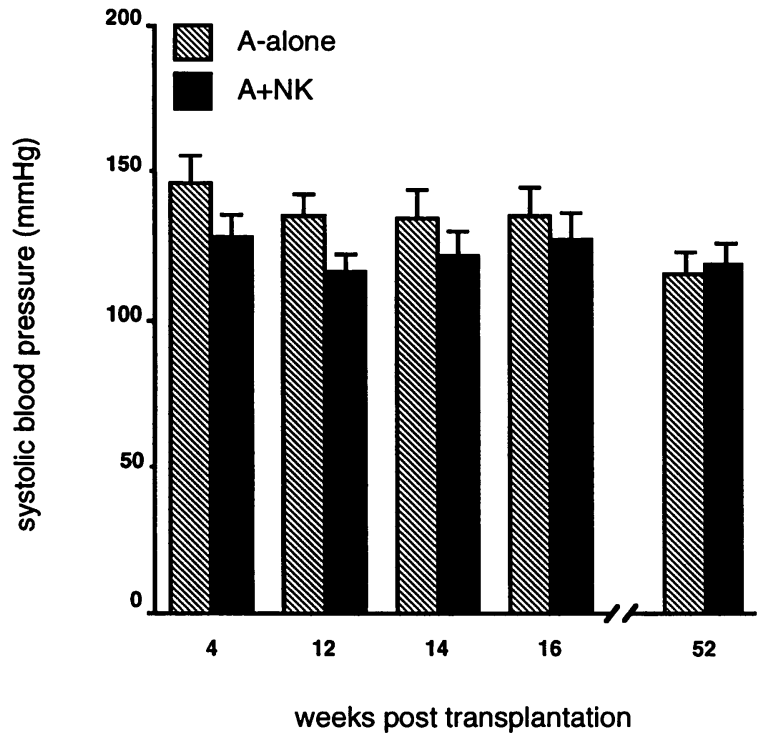

Figure 2. Systolic blood pressure in the solitary allograft group (Aalone, $n=9$ ) is depicted by the shaded columns and from rats in the $\mathrm{A}+\mathrm{NK}$ group $(n=7)$ by the solid columns. While a trend is evident for A-alone SBP to be greater than that for the $A+N K$ rats, at no point does a significant difference between the groups emerge. Data at $52 \mathrm{wk}$ are from separate groups of identically prepared F344 $\rightarrow$ Lew rats.

rats, not significantly different from $378 \pm 23 \mathrm{~g}$ for the A + NK group. As shown in Fig. $3 \mathrm{~A}$, the presence of the native kidney limited the compensatory increases in GFR in the allograft of $A+N K$ rats relative to that seen in the solitary allograft (Aalone). Similarly, effective renal plasma flow rate (ERPF) in A-alone allografts $(5.0 \pm 1.1 \mathrm{ml} / \mathrm{min})$ was far in excess of values for the allograft in the A + NK group $(2.4 \pm 0.5)$, due to the presence of the retained native kidney (ERPF $5.6 \pm 1.4 \mathrm{ml} / \mathrm{min}$ ). In addition to this comparative freedom from hyperfiltration and hyperperfusion, by 16-18 wk the allograft in A + NK rats was also subjected to considerably less compensatory structural hypertrophy, as evident from wet kidney weights determined at the conclusion of the clearance studies (Fig. $3 B$ ), whereas the solitary allografts (A-alone) exhibited marked hypertrophy. In addition to averting compensatory growth and hyperfunction in the allograft, the presence of the native kidney also spared the allograft in A + NK rats from glomerular injury. Only $4 \pm 1 \%$ of glomeruli in the $\mathrm{A}+\mathrm{NK}$ allografts at $18 \mathrm{wk}$ showed evidence of FSGS in comparison with $24 \pm 8 \%$ in the A-alone allografts ( $P<0.05$ by unpaired $t$ test). Furthermore, tubulo-interstitial architecture was well preserved and the intensity of mononuclear interstitial cell infiltrate was minimal in the $\mathrm{A}+\mathrm{NK}$ allograft, and restricted to occasional perivenular areas. In comparison, a moderate, patchy mononuclear cell infiltrate was seen throughout the interstitium of the A-alone allografts. Indeed, the histopathologic appearances of the allografts in A + NK rats were such that, with one exception, they were little different from those of normal kidneys. As shown in Fig. 4, the extent of focal and segmental sclerosis and hyalinosis evident in glomeruli from the solitary allografts (A-alone) showed considerable variation with many showing severe, extensive glomerulosclerosis. Three A-alone rats noted to have a mild degree of allograft hydronephrosis are also included in this figure, although these data points are excluded from computation of the

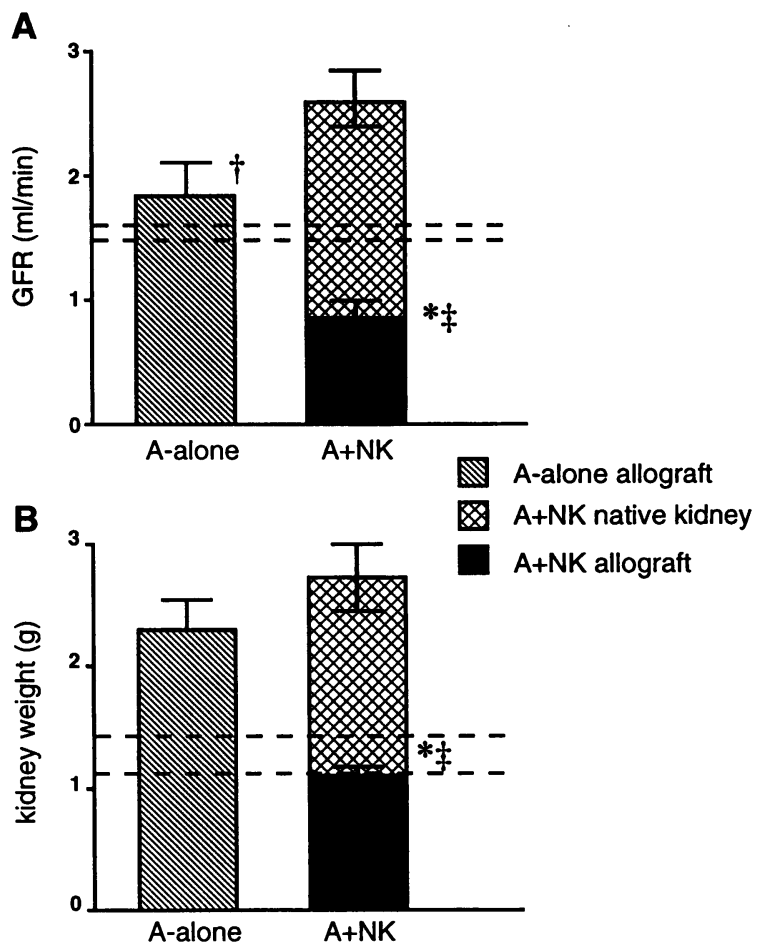

Figure 3. Glomerular filtration rates and kidney weights at $18 \mathrm{wk}$. $(A)$ Mean GFR from A-alone rats $(n=9)$ in the left, hatched column is considerably less than total GFR for A+NK rats $(n=7)$, but appears greater than the range of left kidney GFRs determined under identical conditions in normal, 2-kidney Lewis rats $(n=3)$, as indicated by the horizontal broken lines. Total A+NK GFR is depicted by the right column, divided into allograft (solid) and native kidney (cross-hatched) components. GFR for the allograft in A+NK rats is significantly less than that of the native kidney, and is also less than that of A-alone. ${ }^{*} P$ $<0.05$ vs. A-alone; ${ }^{\dagger} P<0.05$ vs. A + NK (total) $;{ }^{\ddagger} P<0.05$ vs. NK: by single factor analysis of variance and Sheffé's test. $(B)$ Total renal mass was not significantly different between A-alone and A+NK groups. Native kidney weight was significantly greater than allograft weight in $\mathrm{A}+\mathrm{NK}$ rats, and $\mathrm{A}+\mathrm{NK}$ allograft weight was significantly less than that for the A-alone allograft which appeared greater than the range of kidney weights from normal, 2-kidney Lewis rats $(n=3)$, as shown by the horizontal broken lines. ${ }^{*} P<0.05$ vs. A-alone; ${ }^{\ddagger} P<$ 0.05 vs. NK: by single factor analysis of variance and Sheffé's test.

A-alone mean. In two instances, mild hydronephrosis (kidney macroscopically of normal appearance; ureter dilated $<5 \mathrm{~mm}$ proximal to anastomosis) was discernible in vivo at the time of the clearance studies; in the third case, identification was made by the pathologist from the presence of dilated tubules on histological examination. Thus, at least in these three instances, the presence of occult hydronephrosis appeared to be associated with relatively high levels of glomerulosclerosis. Interestingly, the allograft showing the most severe, extensive FSGS $(\approx 90 \%)$ came from a rat in whom marked systemic hypertension ( SBP $>180 \mathrm{mmHg}$ ) was documented 2 wk before acute study. By marked contrast, the native kidneys of $\mathrm{A}+\mathrm{NK}$ rats showed no evidence of glomerular injury whatsoever and were essentially normal in appearance.

\section{Discussion}

The finding that renal mass reduction leads to a process of self-perpetuating renal injury, typified by the histopathologic 


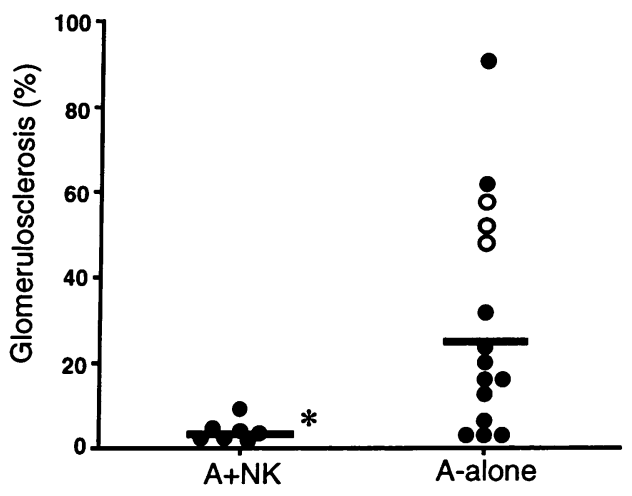

Figure 4. Glomerulosclerosis in individual rats at $18 \mathrm{wk}$. Each data point represents the proportion of glomeruli showing evidence of segmental or global sclerosis in individual rats. Allografts from $\mathrm{A}+\mathrm{NK}$ rats averaged $4 \pm 1 \%$ glomerulosclerosis. By contrast, $A$-alone glomeruli showed greatly elevated levels of glomerulosclerosis, $24 \pm 8 \%$, and considerably grater variation. The open circles depict rats found to have mild hydronephrosis at the time of acute study which were excluded from computation of the mean. ${ }^{*} P<0.05$ by Student's $t$ test for unpaired values.

appearance of focal and segmental glomerulosclerosis and hyalinosis is well established. Although not all are agreed upon the underlying mechanisms, FSGS may be considered as the pathological hallmark of progressive, hemodynamically mediated glomerular injury (10). In this study, we show that the evolution of chronic injury in the F344 $\rightarrow$ Lew renal allograft is, to a large extent, mediated by processes arising as a consequence of inadequate nephron supply. The data also provide the first demonstration that the converse of the renal massreduction paradigm applies, i.e., prevention of progressive renal failure and FSGS may be achieved by renal mass augmentation in a setting where a moderate deficit in nephron supply of somewhat more than $50 \%$ exists. These findings are in accordance with our hypothesis that hemodynamic factors contribute significantly to chronic renal allograft injury. Recent reports in the F344 $\rightarrow$ Lew model provide further support for this view. Measurements of stop-flow pressures in the F344 $\rightarrow$ Lew (solitary) allograft provide estimates of glomerular capillary hydraulic pressure that are clearly elevated with respect either to unoperated F344 controls or to F344 isografts, independent of levels of systemic blood pressure (14). Prolonged study of isografts has revealed that after periods of $1 \mathrm{yr}$, proteinuria and FSGS develop (15), accompanied by profiles of cellular infiltration and cytokine activity similar to those previously documented in the F344 $\rightarrow$ Lew allograft (16). This finding suggests that whereas crossing the immune barrier may advance and accelerate the injury process in allotransplanted rats, perhaps through the initial episode of acute rejection $(4,5)$, the features of the injury processes in allografts, isografts, and remnant kidneys are ultimately quite similar. In support of this possibility, immunohistochemical staining reveals qualitative similarities between the appearances of infiltrating cells and cytokine expression in remnant kidneys and single allografts, irrespective of whether the remnant kidneys were allogeneic, isogeneic, or native in origin, implying that more similarities than diversities exist among the injury processes underlying late failure of allografts, isografts, and remnant kidneys (17).

While identification of the mechanisms underlying the pro- tective effects of renal mass supplementation on the F344 $\rightarrow$ Lew allograft is the subject of further studies underway in our laboratories, the strikingly beneficial effects of augmenting the nephron supply nevertheless prompt the question: to what extent do hemodynamically mediated injury processes contribute to late allograft failure in clinical transplantation? A variety of observations, when taken together, support the notion that in patients, inadequate nephron supply does indeed influence late renal allograft outcome. In humans as in rodents, the greater the degree of ablation of renal mass, the greater the ultimate severity of hypertension, proteinuria, and eventual glomerulosclerosis (12). By analogy, the poorer long-term clinical outcome encountered after long cold-ischemia times (18) or more frequent episodes of acute rejection (19-22), may represent the eventual consequence of fewer and fewer nephrons surviving these early events. Body and kidney size discrepancies between donor and recipient may also be expected to influence the shortfall between the supply of nephrons and the demands placed upon them $(12,21)$; individual differences in body weight, surface area, and kidney weights in humans are highly correlated with numbers of glomeruli present per kidney (22, 23 ). Recent preliminary reports suggest that donor-to-recipient body surface area ratios of $<0.8$ are associated with poorer early and late graft function (24), and that kidney-to-recipient body weight ratios of $<2.5 \mathrm{~g} / \mathrm{kg}$ predict a 5 -yr allograft survival rate of $48 \%$, whereas ratios of $\geq 3.5 \mathrm{~g} / \mathrm{kg}$ are associated with 5-yr survival rates of $71 \%$ (25). Comparison of transplant registry data among demographic groups differing in their renalto-body weight ratios also reveals that the subtle but consistent shortfalls in total renal mass and nephron numbers in females relative to males $(12,26-28)$, and in the US population of African descent relative to Caucasians $(29,30)$, are associated with clear trends for donor kidneys originating from these groups to have poorer survival rates, irrespective of recipient race or gender. The outcome of transplanting kidneys from donors at the extremes of age, where filtration surface area is limited, as in early childhood, or due to age-related glomerulosclerosis, is also worse than for donors of intermediate ages $(31,32)$ although transplantation of both kidneys from pediatric donors may, in some instances at least, abolish this difference (33).

The consistency of these trends, observed across a diversity of histocompatibility factors in clinical transplantation, lends support to the concept that the allograft-to-recipient body weight ratio, and hence the number of nephrons supplied per unit recipient body mass, is an important independent factor influencing late outcome, in keeping with the hypothesis that the adaptive responses of nephrons to inadequate renal mass contribute to the late failure of renal allografts. Since we postulate that the vicious cycle of hemodynamically mediated glomerular injury is "primed" in the allograft, by virtue of initial nephron losses, it may serve as a positive feedback mechanism amplifying the deleterious effects of other factors such as nephron loss through cyclosporine nephrotoxicity or ongoing, alloantigen-specific, immune-mediated injury, which, by themselves, may cause injury or relatively low-grade intensity. By aiming therapeutic intervention to target specifically this positive feedback mechanism, it seems reasonable to anticipate highly effective results, despite the likely multifactorial nature of the processes initiating chronic renal allograft injury. Dietary protein restriction, for example, as reported in some small clinical studies $(34,35)$, or administration of angiotensin I converting enzyme inhibitors, 
as reported for the F344 $\rightarrow$ Lew model (36), may ameliorate or slow the progression of chronic renal allograft failure. The value of matching kidney to donor body size ratios is an additional measure which should clearly also be addressed in clinical studies. Implementation of such relatively inexpensive measures, if indeed proven effective, could have immediate relevance to conservation of function and viability of renal allografts in clinical practice.

In summary, we have demonstrated a substantial protective effect of increasing total nephron supply on indices of chronic allograft injury in the F344 $\rightarrow$ Lew model of late allograft failure. This implies that chronic allograft injury in this model is, to a large extent, associated with processes arising from the renal responses to inadequate nephron supply, and, conversely, that due to permanent losses of nephrons through acute injury, the single allograft often provides an inadequate supply of nephrons with which to meet the metabolic demands of the recipient.

\section{Acknowledgments}

We wish to thank Julia L. Troy, Miguel A. Zayas, and Deborah Sandstrom for expert technical assistance.

This work was supported by U.S. Public Health Service grants 9 R01 DK-461 90-20, DK-35930, and 30410. S.G. Tullius and U.W Heemann were recipients of research fellowships $\mathrm{Tu}$ 63/1-1 and $\mathrm{He}$ 1906/2-1 from the Deutsche Forschungsgemeinschaft.

\section{References}

1. Tilney, N., W. D. Whitley, J. R. Diamond, J. W. Kupiec-Weglinski, and D. H. Adams. 1991. Chronic rejection-an undefined conundrum. Transplantation. 52:389-398.

2. Terasaki, P. I., J. M. Cecka, Y. Cho, J. Cicciarelli, M. Cohn, D. Gjertson, E. Lim, M. R. Mickey, K. Ogura, M. S. Park, S. Takemono, and J. Yuge, 1990. Overview. In Clinical Transplants, 1990. P. I. Terasaki, Editor. UCLA Tissue Typing Lab, Los Angeles. 585-601.

3. Opelz, G., V. Schwartz, A. Engelmann, D. Back, M. Wilk, and E. Keppel. 1991. Long-term impact of HLA matching on kidney graft survival in cyclosporine-treated recipients. Transplant. Proc. 23:373-375.

4. White, E., W. H. Hildemann, and Y. Mullen. 1969. Chronic kidney allograft reactions in rats. Transplantation. 8:602-617.

5. Diamond, J. R., N. L. Tilney, J. Frye, G. Ding, J. McElroy, I. PesekDiamond, and H. Yang. 1992. Progressive albuminuria and glomerulosclerosis in a rat model of chronic renal allograft rejection. Transplantation. 54:710-716.

6. Cheigh, J. S., J. Mouradian, M. Soliman, L. Tapia, R. R. Riggio, K. H. Stenzel, and A. L. Rubin. 1983. Focal segmental glomerulosclerosis in renal transplants. Am. J. Kid. Dis. 2:449-455.

7. Hume, D. M., J. P. Merrill, B. F. Miller, and G. W. Thorn. 1955. Experiences with renal transplantation in the human: report of nine cases. J. Clin. Invest. $34: 327-382$.

8. Porter, K. A., W. B. Thomson, K. Owen, J. R. Kenyon, J. F. Mowbray, and W. S. Peart. 1963. Obliterative changes in four human kidney transplants Br. Med. J. 2:639-645.

9. Brenner, B. M. 1983. Hemodynamically mediated glomerular injury and the progressive nature of glomerular disease. Kidney. Int. 23:647-655.

10. Rennke, H. G., and P. S. Klein. 1989. Pathogenesis and significance of nonprimary focal and segmental glomerulosclerosis. Am. J. Kid. Dis. 13:443456.

11. Hostetter, T. H., J. L. Olson, H. G. Rennke, M. A. Venkatachalam, and B. M. Brenner. 1981. Hyperfiltration in remnant nephrons: a potentially adverse response to renal ablation. Am. J. Physiol. 241:F85-F93.

12. Brenner, B. M., and E. L. Milford. 1993. Nephron underdosing: a programmed cause of chronic renal allograft failure. Am. J. Kid. Dis. 21:66-72.
13. Wallestein, S. C., C. Zucker, and J. L. Fleiss. 1980. Some statistical methods useful in circulation research. Circ Res. 47:1-9.

14. Kingma, I., R. Chea, A. Davidoff, H. Benediktsson, and L. C. Paul. 1993. Glomerular capillary pressures in long-surviving renal allografts. Transplantation. 56:53-59.

15. Tullius, S. G., U. Heemann, W. W. Hancock, H. Azuma, and N. L. Tilney. 1993. Isografts develop functional and structural changes which mimic those of chronic allograft rejection. J. Am. Soc. Nephrol. 4:920 (Abstract).

16. Hancock, W. W., W. D. Whitley, S. G. Tullius, U. W. Heemann, B. Wasowska, W. M. Baldwin, and N. L. Tilney 1993. Cytokines, adhesion molecules and the pathogenesis of chronic rejection of rat renal allografts. Transplantation. $56: 643-650$

17. Heemann, U. W., H. Azuma, S. G. Tullius, and N. L. Tilney. 1993. The influence of reduced kidney mass on chronic kidney rejection in rats. J. Am. Soc. Nephrol. 432:912 (Abstract)

18. Foster, M. C., P. A. Rowe, M. J. S. Dennis, A. G. Morgan, R. P. Burden, and R. W. Blamey. 1990. Characteristics of cadaveric renal allograft recipients developing chronic rejection. Ann. R. Coll. Surg. Eng. 72:23-26.

19. Almond, P. S., A. Matas, K. Gillingham, D. L. Dunn, W. D. Payne, P. Gores, R. Gruessner, and J. S. Najarian. 1993. Risk factors for chronic rejection in renal allograft recipients. Transplantation. 55:752-757.

20. Basadonna, G. P., A. J. Matas, K. J. Gillingham, W. D. Payne, D. L. Dunn, D. E. R. Sutherland, P. F. Gores, R. W. G. Gruessner, and J. S. Najarian. 1993. Early versus late acute renal allograft rejection: impact on chronic rejection. Transplantation. 55:993-995.

21. Cecka, J. M. 1988. Donor and preservation factors. In Clinical Transplants, 1988. P. I. Terasaki, Editor. UCLA Tisue Typing Lab, Los Angeles. 428-430.

22. Kasiske, B. L., and A. J. Umen. 1986. The influence of age, sex, race and body habitus on kidney weight in humans. Arch. Pathol. Biol. Med. 110:55-60.

23. Nyengaard, J. R., and T. F. Bendtsen. 1992. Glomerular number and size in relation to age, kidney weight, and body surface area in normal man. Anat. Rec. 232:194-201

24. Kupin, W. L., K. K. Venkat and M. Mozes. 1993. Predictive value of the donor surface area / recipient surface area ratioo (DSA/RSA) on the outcome of primary cadaveric renal transplantation. J. Am. Soc. Nephrol. 4:945 (Abstr).

25. Roth, D., G. Burke, J. Nery, V. Esquenazi, and J. Miller. 1994. Nephron dosing in renal transplantation: How much kidney is sufficient? Abstracts of the American Society of Transplant Physicians 13th Annual Meeting . Chicago, IL. 1994:50A (Abstract).

26. McLachlan, M. S. F., J. C. Guthrie, C. K. Anderson, and M. J. Fuller. 1977. Vascular and glomerular changes in the ageing kidney. J. Pathol. 121:6578.

27. Mickey, R., Y. W. Cho, and E. Carnahan. 1990. Long-term graft survival. In Clinical Transplants, 1990. P. I. Terasaki, Editor. UCLA Tissue Typing Lab, Los Angeles. 385-396.

28. Terasaki, P. I., J. M. Cecka, S. Takemoto, and J. Yuge. 1988. Overview. In Clinical Transplants, 1988. P. I. Terasaki, Editor. UCLA Tissue Typing Lab, Los Angeles. 428-430.

29. Brenner, B. M., R. A. Cohen, and E. L. Milford. 1992. In renal transplantation one size may not fit all. J. Am. Soc. Nephrol. 3:162-169.

30. Zhou, Y. C., P. I. Terasaki, and J. M. Cecka. 1990. Race effects on the outcome of kidney transplants. In Clinical Transplants, 1990. P. I. Terasaki, Editor. UCLA Tissue Typing Lab, Los Angeles. 447-459.

31. Hayes, J. M., D. R. Steinmuller, and S. B. S. C. Novick. 1991. The development of proteinuria and focal segmental glomerulosclerosis in recipients of pediatric donor kidneys. Transplantation. 52:813-817.

32. Alexander, J. W., and W. Vaughn. 1991. The use of "marginal" donors for organ transplantation. The influence of donor age on outcome. Transplantation. 51:135-141.

33. Sutherland, D. E. R., R. W. G. Gruessner, A. J. Matas, G. Lloveras, D. S. Fryd, D. L. Dunn, W. D. Payne, and J. S. Najarian. 1991. Transplantation of single and double kidneys from pediatric donors. In Organ Transplantation 1990. G. M. Abouna, M. S. A. Kumar and A. G. White, editors. Kluwer Academic Publication, Dordrecht.

34. Feehally, J., K. P. G. Harris, S. E. Bennett, and J. Walls. 1986. Is chronic rejection a non-immunological phenomenon? Lancet. 2:486-488.

35. Salahudeen, A. K., T. H. Hostetter, S. K. Raatz, and M. E. Rosenberg. 1992. Effects of dietary protein in patients with chronic renal transplant rejection. Kidney Int. 41:183-190.

36. Paul, L. C., R. Chea, A. Davidoff, and H. Bendiktsson. 1993. Hemodynamic factors in transplant glomerulopathy in the rat. J. Am. Soc. Nephrol. 4:917 (Abstract). 